\title{
Óxido de cromo e n-alcanos na estimativa do consumo de forragem de vacas em lactação em condições de pastejo ${ }^{1}$
}

\author{
Mirton José Frota Morenz ${ }^{2}$, José Fernando Coelho da Silva ${ }^{3}$, Luiz Januário Magalhães \\ Aroeira ${ }^{4}$, Fermino Deresz ${ }^{4}$, Hernan Maldonado Vásquez ${ }^{3}$, Domingos Sávio Campos Paciullo ${ }^{4}$, \\ Fernando César Ferraz Lopes ${ }^{4}$, Ana Cristina Wyllie Elyas ${ }^{5}$, Edenio Detmann ${ }^{6}$

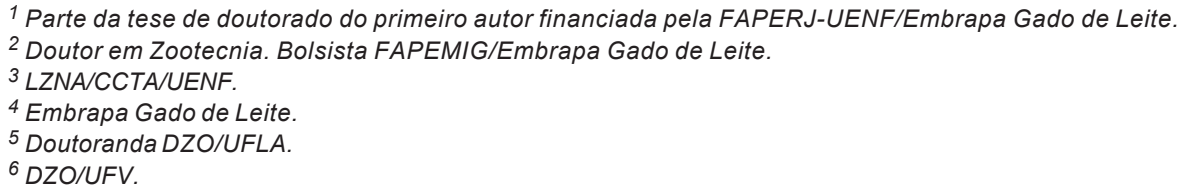

RESUMO - Foram avaliadas as técnicas do óxido de cromo/digestibilidade in vitro e dos pares de $\mathrm{n}$-alcanos $\left(\mathrm{C}_{31}\right.$ : $\mathrm{C}_{32}$ e $\mathrm{C}_{33}: \mathrm{C}_{32}$ ) na estimativa do consumo de matéria seca (CMS) por vacas Holandês x Zebu em lactação em pastagem de capimelefante cv. Napier. As extrusas foram obtidas utilizando-se uma vaca com fístula esofágica. Realizaram-se coletas de fezes duas vezes ao dia, diretamente no reto dos animais, durante nove dias em cada período experimental. O delineamento utilizado foi o de blocos casualizados, em esquema de parcelas divididas. Ambos os pares de n-alcanos possibilitaram estimativas de consumos semelhantes, independentemente do horário de coleta (manhã e tarde), sugerindo que uma única coleta de fezes por dia seria suficiente. Quando o estudo foi realizado utilizando-se o valor médio das duas coletas, todas as metodologias diferiram entre si. $\mathrm{O} \mathrm{Cr}_{2} \mathrm{O}_{3}$ /DIVMS forneceu valores de CMS que podem ser considerados mais adequados, pois foram mais próximos daqueles estimados para forragem consumível (2,6\% PV) e dos valores das tabelas de exigências do NRC (2001) $(2,7 \% \mathrm{PV})$. Além disso, essa técnica apresentou vantagens quanto à simplicidade dos procedimentos analíticos e ao baixo custo.

Palavras-chave: consumo de matéria seca, métodos indiretos, pastejo rotacionado, Pennisetum purpureum

\section{Use of chromium oxide or n-alkanes to estimate forage intake of grazing lactating cows}

\begin{abstract}
Chromium oxide and n-alkanes $\left(\mathrm{C}_{31}: \mathrm{C}_{32}\right.$ and $\left.\mathrm{C}_{33}: \mathrm{C}_{32}\right)$ were used to estimate the dry matter intake (DMI) of lactating Holstein x Zebu cows grazing elephant grass. Extrusa samples of forage were obtained from a cow with esophageal fistula. Fecal grab collection was done twice a day at 7:30 and 14:30 h during 9 days in each experimental period. A randomized complete block design with a split-plot arrangement was used. Both pairs of n-alkanes estimated similar DMI, independent of time of fecal collection, suggesting that one single daily collection of feces may be adequate. Independent of the fecal collection schedule, DMI estimated by $\mathrm{Cr}_{2} \mathrm{O}_{3}$ or n-alkanes differed significantly by the Tukey test. Dry matter intake estimated by $\mathrm{Cr}_{2} \mathrm{O}_{3}$ was closer to that predicted according to the available forage ( $2.6 \%$ body weight, $\mathrm{BW}$ ) and the NRC (2001) model $(2.7 \% \mathrm{BW})$. Therefore, DMI estimated by $\mathrm{Cr}_{2} \mathrm{O}_{3}$ is likely to be more accurate than n-alkanes. In addition, $\mathrm{Cr}_{2} \mathrm{O}_{3}$ is inexpensive and easy to analyze.
\end{abstract}

Key Words: dry matter intake, indirect methods, Pennisetum purpureum, rotational grazing

\section{Introdução}

O sistema de criação do rebanho bovino nacional tem como base a utilização de pastagens como principal fonte de nutrientes para os animais. As vantagens econômicas da utilização de pastagens como fonte primária de energia na dieta de vacas leiteiras são evidentes, mas tem-se como limitação o conhecimento do quanto das exigências do animal são atendidas pela forragem ingerida, essencial para a formulação de dietas e a adoção de estratégias de alimentação adequadas, que possibilitem a avaliação do desempenho do rebanho, ajustando as condições de produção de acordo com a qualidade e a disponibilidade de forragem, tornando a atividade mais econômica e produtiva.

A estimativa acurada do CMS por animais criados em pastejo sempre foi um desafio para os pesquisadores, tendo vista o grande número de variáveis que atuam no controle 
do consumo e as limitações impostas pelas metodologias utilizadas para obtenção destas estimativas.

A metodologia do óxido de cromo $\left(\mathrm{Cr}_{2} \mathrm{O}_{3}\right)$, associada à digestibilidade in vitro da matéria seca (DIVMS), tem sido amplamente utilizada na obtenção das estimativas de CMS de animais em pastejo (Malossini et al., 1996; Aroeira, 1997; Astigarraga, 1997). Entretanto, possui desvantagens como a interferência direta no comportamento ingestivo do animal (Aroeira, 1997), o fornecimento de um único valor de digestibilidade para o grupo de animais (Berry et al., 2000; Gedir \& Hudson, 2000), além dos problemas referentes à recuperação fecal do indicador (Malossini et al., 1996).

A técnica de n-alcanos (Mayes et al., 1986; Dove \& Mayes, 1991; Malossini et al., 1996) baseia-se na combinação de um n-alcano de cadeia com número ímpar de carbonos, naturalmente encontrado nas plantas, com um n-alcano sintético, de cadeia com número par de carbonos, utilizado como indicador externo. Segundo Gedir \& Hudson (2000), os n-alcanos mais viáveis como indicadores externos são o n-dotriacontano $\left(\mathrm{C}_{32}\right)$ e o n-hexatriacontano $\left(\mathrm{C}_{36}\right)$, em razão do menor custo, da fácil obtenção na forma pura e por apresentar-se em baixas concentrações nas plantas. O n-alcano de cadeia ímpar, para ser utilizado como indicador interno de digestibilidade da MS, deveria estar presente na forragem em concentração mínima de $50 \mathrm{mg} / \mathrm{kg}$ MS (Laredo et al., 1991).

A estimativa do consumo obtida pela técnica de nalcanos não é influenciada pela digestibilidade individual da ração ou pela recuperação dos indicadores, desde que as recuperações fecais dos n-alcanos de cadeia ímpar (contidos na forragem) e daqueles de cadeia par (fornecidos oralmente) sejam similares (Dove \& Mayes, 1991; Malossini et al., 1996). Além disso, quando administrado por meio de cápsulas de liberação controlada (Controlled Release Capsules-CRC), faz-se uma única aplicação no primeiro dia do experimento, o que permite a redução do estresse nos animais durante a aplicação do indicador.

De acordo com a literatura, o par $\mathrm{C}_{33}: \mathrm{C}_{32}$ é o mais indicado, em detrimento dos demais pares, na estimativa do consumo de forragem (Reeves et al., 1996; Unal et al., 1997; Berry et al., 2000; Gedir \& Hudson, 2000; Oliveira, 2003; Molina et al., 2004).

O objetivo neste trabalho foi avaliar as metodologias do óxido de cromo/digestibilidade in vitro e dos n-alcanos $\mathrm{C}_{31}, \mathrm{C}_{32}$ e $\mathrm{C}_{33}$ na estimativa de consumo de MS por vacas Holandês x Zebu em lactação em pastagem de capim-elefante cv. Napier.

\section{Material e Métodos}

O experimento foi realizado durante os meses de março e abril de 2002 no Campo Experimental de Coronel Pacheco da Embrapa Gado de Leite, em Coronel Pacheco, MG.

A pastagem de capim-elefante (Pennisetum purpureum Schum, cv. Napier) foi adubada nos últimos dez anos com $1.000 \mathrm{~kg} / \mathrm{ha} /$ ano da fórmula 20-05-20. O adubo foi distribuído em três parcelas iguais nos meses de novembro, janeiro e março.

A massa de forragem disponível foi estimada em cada período experimental, adotando-se o método descrito por Cóser et al. (2003): um dia antes da entrada dos animais nos piquetes, foram selecionadas três touceiras de capimelefante representativas daquelas encontradas no piquete, correspondentes às produções de forragem das touceiras com disponibilidade alta, média e baixa. As amostragens foram feitas manualmente e toda a parte aérea (folhas e colmos tenros) foi coletada, pesada e levada à estufa para determinação do teor de MS. A média do peso seco das três touceiras, multiplicada pelo número de touceiras contadas em $49 \mathrm{~m}^{2}$ de cada piquete, foi utilizada para estimativa da massa $(\mathrm{kg} / \mathrm{ha})$ e da oferta $(\mathrm{kg} / \mathrm{animal} / \mathrm{dia})$ de MS de forragem.

Foram utilizadas oito vacas Holandês x Zebu em lactação, com $472 \pm 49$ e $477 \pm 49 \mathrm{~kg}, 65 \pm 19,7$ e $66 \pm 19,7$ meses de idade e $124 \pm 33,8$ e $154 \pm 33,8$ dias em lactação, no primeiro e segundo períodos experimentais, respectivamente. Os animais foram pesados antes e após cada período experimental, sendo ordenhados mecanicamente, duas vezes ao dia (6 e 14h). As produções de leite foram de 10,4 $\pm 2,6$ e 9,6 $\pm 2,5 \mathrm{~kg} / \mathrm{dia}$, no primeiro e segundo períodos experimentais, respectivamente.

Os animais receberam como suplemento apenas sal mineral e foram mantidos em uma área de pastagem exclusiva de capim-elefante, manejada em sistema rotacionado, com ciclo de pastejo de 33 dias (30 de descanso e três de ocupação).

As amostras de extrusa foram obtidas de uma vaca Holandês x Zebu (peso médio de $540 \mathrm{~kg}$ ) com fístula esofágica, em jejum de 12 horas. O conteúdo desvido do esôfago (Van Dyne \& Torrel, 1964) foi coletado utilizando-se bolsa de lona com tela plástica rígida no fundo para facilitar a drenagem de saliva.

As coletas de extrusa foram realizadas durante nove dias consecutivos, no 1ํㅜ $2^{\circ}$ e $3 \stackrel{\circ}{\circ}$ dias de ocupação de cada um dos três piquetes, em cada período experimental, durante aproximadamente 30 minutos de pastejo, pela manhã. Imediatamente após a coleta, as amostras de extrusa foram 
congeladas a, aproximadamente, $-18^{\circ} \mathrm{C}$. Ao final do período experimental, foram descongeladas, pré-secas em estufas de ventilação forçada a $60^{\circ} \mathrm{C}\left( \pm 5^{\circ} \mathrm{C}\right)$, durante 72 horas, processadas em moinho tipo Willey com peneira de $1 \mathrm{~mm}$ e analisadas para obtenção do teor de MS. Para estimativa da DIVMS (Tilley \& Terry, 1963), foi utilizada uma amostra representativa de cada período, constituída pelas extrusas do $1^{1}, 2^{\underline{0}}$ e $3 \underline{0}$ dias de ocupação de cada piquete (repetição).

As amostras de fezes foram coletadas diretamente do reto dos animais, durante nove dias, duas vezes ao dia ( $7 \mathrm{~h} 30$ e $14 \mathrm{~h} 30$ ), e imediatamente congeladas a $-18^{\circ} \mathrm{C}$. Ao final do experimento, foram descongeladas, pré-secas em estufa de ventilação forçada, a $60^{\circ} \mathrm{C}\left( \pm 5^{\circ} \mathrm{C}\right)$, durante 72 horas, trituradas em moinho tipo Willey com peneira de $1 \mathrm{~mm}$ e analisadas para determinação do teor de MS.

Para determinação do teor de $\mathrm{Cr}_{2} \mathrm{O}_{3}$ e dos n-alcanos (natural e sintético), foram utilizadas amostras compostas por turno (manhã e tarde), constituídas dos nove dias de coleta, em cada animal, para cada período.

$\mathrm{O} \mathrm{Cr}_{2} \mathrm{O}_{3}$ foi administrado via oral, em doses de $5 \mathrm{~g}$, duas vezes ao dia (7h30 e 14h30), embalado em "papel-toalha". O procedimento foi realizado durante 14 dias: nos cinco primeiros dias, foram obtidos o equilíbrio da ingestão e a excreção do indicador e, a partir do sexto dia, foram feitas as coletas de fezes.

O CMS foi estimado utilizando-se a fórmula: $\mathrm{CMS}=$ produção fecal/(1 - DIVMS) e a produção fecal (PF), em kg de MS/dia, por meio da seguinte fórmula:

$\mathrm{PF}=$ cromo administrado $(\mathrm{g} / \mathrm{dia}) /$ cromo nas fezes $(\mathrm{g} / \mathrm{kg}$ de MS) (Pond et al., 1989).

O teor de cromo nas fezes foi determinado com auxílio de espectrofotômetro de absorção atômica, conforme metodologia descrita por Williams et al. (1962).

Os n-alcanos foram administrados em forma de cápsulas de liberação controlada (CRC, type MCM, Captec Ltd, Auckland, New Zealand) contendo n-dotriacontano $\left(\mathrm{C}_{32}\right)$ e n-hexatriacontano $\left(\mathrm{C}_{36}\right)$, desenvolvidas para uso em bovinos com peso de $300 \mathrm{a} 650 \mathrm{~kg}$. Segundo o fabricante, o tempo total de liberação dos n-alcanos no interior do rúmen é de, aproximadamente, 20 dias a uma taxa constante de 397,2 e 401,3 mg/dia para os n-alcanos $C_{32}$ e $C_{36}$, respectivamente.

A aplicação das cápsulas CRC foi feita (uma única vez) após a ordenha da manhã, no $1^{\circ}$ dia do experimento; nos primeiros sete dias, foram obtidos o equilíbrio da ingestão e a excreção do indicador e, a partir do $8^{\circ} \mathrm{dia}$, foram feitas as coletas de fezes, durante nove dias, em cada período (março e abril). Foram coletadas diariamente duas amostras individuais de fezes ( $7 \mathrm{~h} 30$ e 14h30), que foram analisadas separadamente quanto aos teores de cromo e n-alcanos. Nas amostras de extrusas e de fezes, foram determinadas as concentrações dos n-alcanos $\mathrm{C}_{27}$ a $\mathrm{C}_{35}$, utilizando-se o $\mathrm{C}_{34}$ como padrão interno, segundo protocolo proposto por Oliveira (2004), e o método de cromatografia gasosa. Para o cálculo do consumo de forragem ( $\mathrm{kg} \mathrm{de} \mathrm{MS/dia),} \mathrm{adotou-se}$ a equação:

$$
\mathrm{CMS}=\mathrm{F}_{\mathrm{i}} / \mathrm{F}_{\mathrm{j}}{ }^{*} \mathrm{D}_{\mathrm{j}} / \mathrm{H}_{\mathrm{i}}-\mathrm{F}_{\mathrm{i}} / \mathrm{F}_{\mathrm{j}} * \mathrm{H}_{\mathrm{j}} \text { (Mayes et al., 1986), }
$$

em que $\mathrm{Fi}$ = concentração do n-alcano de cadeia ímpar nas fezes (mg/kg de MS); Fj = concentração do n-alcano de cadeia par nas fezes ( $\mathrm{mg} / \mathrm{kg}$ de $\mathrm{MS}) ; \mathrm{Dj}=$ quantidade do n-alcano sintético fornecido (mg/dia); Hi = concentração do n-alcano de cadeia ímpar natural na forragem $(\mathrm{mg} / \mathrm{kg}$ de MS); $\mathrm{Hj}=$ concentração do n-alcano de cadeia par na forragem ( $\mathrm{mg} / \mathrm{kg}$ de MS).

As estimativas de ingestão de MS foram realizadas utilizando-se dois pares de n-alcanos $\left(\mathrm{C}_{31}: \mathrm{C}_{32}\right.$ e $\left.\mathrm{C}_{33}: \mathrm{C}_{32}\right)$ e a metodologia do $\mathrm{Cr}_{2} \mathrm{O}_{3}$ /digestibilidade in vitro.

No estudo do efeito das metodologias na estimativa do consumo em diferentes turnos de coleta de fezes, empregou-se o delineamento em blocos casualizados, com 16 repetições (sub-blocos), em que cada sub-bloco foi composto pela interação animal x período, em um esquema de parcelas divididas, em que a parcela principal foi composta pelos indicadores e as subparcelas, pelos turnos de coleta. Para avaliação das metodologias com base nos valores médios das duas coletas, utilizou-se o delineamento em blocos, com 16 repetições (sub-blocos). As médias foram comparadas pelo teste Tukey a $5 \%$ de probabilidade e os resultados foram interpretados por meio do software SAEG(UFV, 2000).

\section{Resultados e Discussão}

Em ordem decrescente, os n-alcanos encontrados em maiores concentrações foram o $\mathrm{C}_{29}, \mathrm{C}_{33}$ e $\mathrm{C}_{31}$ (Tabela 1). Foram observadas concentrações superiores para os n-alcanos de cadeia ímpar em relação aos de cadeia par, o que está de acordo com os dados da literatura (Dove \& Mayes, 1996; Oliveira et al., 1997; Lopes et al., 2001; Oliveira, 2003).

Os valores médios estimados de massa de forragem consumível foram de $1.856 \pm 459$ e $1.892 \pm 339 \mathrm{~kg} / \mathrm{ha}$ de MS, que correspondem a uma oferta de MS de 12,4 $\pm 3,1$ e 12,6 $6 \pm 2,3$ $\mathrm{kg} / \mathrm{vaca} / \mathrm{dia}$ ou $2,6 \pm 0,7$ e $2,6 \pm 0,5 \% \mathrm{PV}$, respectivamente, para os meses de março e abril. Estes valores são semelhantes aos relatados por Cóser et al. (2003) para os meses de março e abril de 2001 (1.754 $\pm 404,2$ e $2.027 \pm 407,2 \mathrm{~kg} / \mathrm{ha} \mathrm{de}$ 
Tabela 1 - Concentração de n-alcanos na extrusa do capim-elefante cv. Napier Table 1 - Concentration of $n$-alkanes on extrusa of cows grazing elephantgrass $\mathrm{cv}$. Napier

\begin{tabular}{|c|c|c|c|c|c|c|c|c|c|}
\hline \multirow[b]{2}{*}{$\begin{array}{l}\text { Mês } \\
\text { Month }\end{array}$} & \multicolumn{9}{|c|}{$\begin{array}{c}\mathrm{n} \text {-alcanos }(\mathrm{mg} / \mathrm{kg} \text { de matéria seca) } \\
\text { n-alkanes (mg/kg of dry matter) }\end{array}$} \\
\hline & $\mathrm{C}_{27}$ & $\mathrm{C}_{28}$ & $\mathrm{C}_{29}$ & $\mathrm{C}_{30}$ & $\mathrm{C}_{31}$ & $\mathrm{C}_{32}$ & $\mathrm{C}_{33}$ & $\mathrm{C}_{35}$ & $\mathrm{C}_{36}$ \\
\hline $\begin{array}{l}\text { Março } \\
\text { March }\end{array}$ & 23,3 & 18,1 & 200,9 & $\operatorname{tr}$ & 120,1 & 6,9 & 152,2 & 58,8 & $\operatorname{tr}$ \\
\hline $\begin{array}{l}\text { Abril } \\
\text { April }\end{array}$ & 35,0 & 19,1 & 205,9 & 7,6 & 113,0 & 6,6 & 139,4 & 55,3 & $\operatorname{tr}$ \\
\hline
\end{tabular}

$\operatorname{tr}=\operatorname{traço~}(\operatorname{tr}=$ trace $)$.

MS), por Lopes et al. (2003) para o mês de abril de 1994 (1.617 $\mathrm{kg} / \mathrm{ha}$ de MS) e por Paim-Costa et al. (2004) para o mês de março $(2.100 \mathrm{~kg} / \mathrm{ha}$ de MS), para o capim-elefante manejado sob pastejo rotacionado (três dias de ocupação dos piquetes e 30 dias de descanso).

Os valores estimados de CMS variaram conforme a metodologia, sendo observada interação dos fatores $(\mathrm{P}<0,01)$. As médias estimadas de consumo e suas comparações encontram-se na Tabela 2.

No estudo das interações dos turnos de coleta para cada metodologia, verificou-se diferença $(\mathrm{P}<0,05)$ apenas para o óxido de cromo. Ambos os pares de n-alcanos possibilitaram estimar consumos semelhantes $(\mathrm{P}>0,05)$, independentemente do horário de coleta, evidenciando que uma única coleta de fezes por dia seria suficiente para obtenção de valores confiáveis.

Reeves et al. (1995) também verificaram que amostras fecais coletadas pela manhã e à tarde permitiram estimativas de consumo estatisticamente semelhantes.

Malossini et al. (1996) compararam as duas metodologias de estimativa de consumo utilizando vacas lactantes em pastagem e observaram maiores variações das concentrações fecais de $\mathrm{Cr}_{2} \mathrm{O}_{3}$ ao longo do dia, quando comparadas às dos n-alcanos (pares $\mathrm{C}_{31}: \mathrm{C}_{32} \mathrm{e} \mathrm{C}_{33}: \mathrm{C}_{32}$ ), o que também evidencia a possibilidade de redução do número de coleta de fezes pela técnica dos n-alcanos.

A possibilidade de redução do número de coletas de fezes pela metodologia de $n$-alcanos pode contribuir para minimizar o estresse causado pelos procedimentos de contenção dos animais, fornecendo resultados mais acurados. Além disso, quando são utilizadas cápsulas de liberação controlada (CRC), administradas uma única vez no primeiro dia do período experimental, a interferência no comportamento do animal é ainda menor.

No estudo da interação das metodologias de estimativa do consumo dentro de cada turno, observaram-se, na coleta da manhã, valores de consumo semelhantes $(\mathrm{P}>0,05)$ para o óxido de cromo e para o par $\mathrm{C}_{33}: \mathrm{C}_{32}$, diferindo $(\mathrm{P}<0,05)$, no entanto, do par $\mathrm{C}_{31}: \mathrm{C}_{32}$. Para as amostras coletadas no turno da manhã, os pares de n-alcanos não diferiram $(\mathrm{P}>0,05)$ entre si na estimativa do CMS. Na coleta da tarde (14h30), foram observadas diferentes $(\mathrm{P}<0,05)$ estimativas de CMS entre as metodologias. Em valores absolutos, nos dois turnos, a metodologia do óxido de cromo/DIVMS e o par de n-alcanos $\mathrm{C}_{31}: \mathrm{C}_{32}$ estimaram sempre os maiores e os menores valores de CMS, respectivamente.

Utilizando-se a média das estimativas de CMS provenientes das duas coletas diárias de fezes, todas as metodologias diferiram $(\mathrm{P}<0,01)$ entre si (Tabela 3$)$.

A metodologia do $\mathrm{Cr}_{2} \mathrm{O}_{3}$ /DIVMS forneceu valores de consumo mais próximos aos estimados para a forragem consumível (2,6\% PV) e dos valores das tabelas de exigências do National Research Council (NRC, 2001) - 2,7\% PV (valor tabelado). Os valores de CMS estimados por esta técnica foram, também, mais precisos, visto que os indicadores de dispersão (coeficiente de variação - CV e erropadrão - EP) (Tabela 3) foram inferiores aos obtidos com a metodologia dos n-alcanos.

Euclides et al. (2000) utilizaram a técnica do $\mathrm{Cr}_{2} \mathrm{O}_{3} /$ DIVMS na estimativa do CMS dos capins Brachiaria brizantha cv. Marandu e B. decumbens por novilhos Nelore no período das águas e encontraram valores de 2,79 e 2,67\% $\mathrm{PV}$, respectivamente. Em vacas em lactação em pastagem de capim-elefante manejada com 30 dias de descanso no período das chuvas, Paciullo et al. (2001) obtiveram valores de CMS de 2,5\% PV pela metodologia do $\mathrm{Cr}_{2} \mathrm{O}_{3}$ /DIVMS inferiores àqueles encontrados por Lopes et al. (2004), pelo método $\mathrm{Cr}_{2} \mathrm{O}_{3}$ /DIVMS, em vacas Holandês $x$ Zebu em pastagem de capim-elefante com 30 dias de descanso no período das águas $(2,9 \%$ PV, em média).

Considerando que, em condições de pastejo, o consumo verdadeiro não é conhecido, os valores estimados pelos n-alcanos só podem ser comparados aos obtidos a partir de outros métodos, que também possuem "vícios".

Dados na literatura demonstram a tendência de o $\mathrm{Cr}_{2} \mathrm{O}_{3}$ superestimar o consumo (Piasentier et al., 1995; Malossini et al., 1996; Detmann et al., 2001). A metodologia do $\mathrm{Cr}_{2} \mathrm{O}_{3}$ / DIVMS possibilitou estimar CMS 12,4\% superior ao obtido 
Tabela 2 - Médias e erros-padrão da média de CMS (\%PV) de forragem de capim-elefante, estimados por diferentes metodologias em dois turnos de coleta (manhã e tarde) ${ }^{1}$

Table 2 - Means and standard errors of means for daily dry matter intake (\% body weight, $\%$ BW) of elephantgrass estimated by $\mathrm{Cr}_{2} \mathrm{O}_{3}$ or $n$-alkanes at different times of fecal collection (morning and afternoon)

Turno de coleta

Collection schedule
Metodologia

Marker

\begin{tabular}{ccc}
\hline $\mathrm{Cr}_{2} \mathrm{O}_{3} / \mathrm{DIVMS}$ & $\mathrm{C}_{31}: \mathrm{C}_{32}$ n-alcanos & $\mathrm{C}_{33}: \mathrm{C}_{32}$ n-alcanos \\
$\mathrm{Cr}_{2} \mathrm{O}_{3} /$ IVDMD & $\mathrm{C}_{31}: \mathrm{C}_{32}{ }^{\text {n-alkanes }}$ & $\mathrm{C}_{33}: \mathrm{C}_{32}{ }^{\text {n-alkanes }}$ \\
\hline $2,33 \pm 0,063 \mathrm{Ba}$ & $1,970,085 \mathrm{Ab}$ & $2,17 \pm 0,093 \mathrm{Aab}$ \\
$2,56 \pm 0,098 \mathrm{Aa}$ & $1,89 \pm 0,091 \mathrm{Ac}$ & $2,19 \pm 0,098 \mathrm{Ab}$
\end{tabular}

Afternoon

${ }^{1}$ Médias seguidas das mesmas letras maiúsculas nas colunas e minúsculas nas linhas não diferem pelos testes $F(P>0,05)$ e Tukey $(P>0,05)$, respectivamente.

${ }^{1}$ Means followed by same capital letters within columns and small letters within rows, do not differ by $F(P>.05)$ and Tukey $(P>.05)$ tests, respectively.

Tabela 3 - Médias, coeficientes de variação (CV) e errospadrão da média (EP) do CMS (\%PV) de pastagem de capim-elefante, estimados por diferentes metodologias, considerando o valor médio de duas coletas de fezes ${ }^{1}$

Tabela 3 - Means, coefficients of variation (CV) and standard errors of mean (SE) for daily dry matter intake (\%BW) of elephantgrass estimated by $\mathrm{Cr}_{2} \mathrm{O}_{3}$ or $n$-alkanes using the mean value of the two fecal collection

\begin{tabular}{lccc}
\hline $\begin{array}{l}\text { Metodologia } \\
\text { Marker }\end{array}$ & CMS (\% PV) & $\mathrm{CV}(\%)$ & $\mathrm{EP}$ \\
& $D M I(\% \mathrm{BW})$ & & $S E$ \\
\hline $\mathrm{Cr}_{2} \mathrm{O}_{3}$ & $2,45 \mathrm{a}$ & 14,08 & 0,061 \\
$\mathrm{C}_{31}: \mathrm{C}_{32}$ & $1,93 \mathrm{~b}$ & 18,14 & 0,062 \\
$\mathrm{C}_{33}: \mathrm{C}_{32}$ & $2,18 \mathrm{c}$ & 17,22 & 0,066
\end{tabular}

${ }^{1}$ Médias seguidas das mesmas letras não diferem $(P>0,01)$ pelo teste Tukey.

${ }^{1}$ Means followed by same letters do not differ $(P>0.01)$ by Tukey test.

com o par de n-alcanos $\mathrm{C}_{33}: \mathrm{C}_{32}$ e $26,9 \%$ superior ao estimado pelo par $\mathrm{C}_{31}: \mathrm{C}_{32}$. Avaliando a técnica do $\mathrm{Cr}_{2} \mathrm{O}_{3}$ /DIVMS na estimativa do CMS em vacas em lactação, Soares et al. (1999; 2004) observaram que o consumo real, medido em cochos do tipo calan-gates, foi superestimado em 13,9\% pelo $\mathrm{Cr}_{2} \mathrm{O}_{3}$ /DIVMS (Soares et al., 1999) e 9,3\% (Soares et al., 2004). Estes autores atribuíram esta superestimativa à recuperação incompleta do indicador $\left(\mathrm{Cr}_{2} \mathrm{O}_{3}\right)$ nas fezes. No entanto, Fontes et al. (1996), em novilhos, observaram que as recuperações fecais do óxido de cromo foram, em média, $103,3 \%$.

Além dos problemas relativos à recuperação do $\mathrm{Cr}_{2} \mathrm{O}_{3}$ nas fezes, os valores de DIVMS obtidos foram superiores aos estimados com os n-alcanos $\mathrm{C}_{31} \mathrm{e}_{33}$, utilizados como indicadores internos. Esse fato, adicionalmente, pode ter contribuído para as diferenças observadas nos consumos estimados por estas metodologias. De forma semelhante, Detmann et al. (2001) relataram valores de DIVMS (Tilley \& Terry, 1963) superiores aos determinados com o uso de outros indicadores internos de digestibilidade.
A tendência da metodologia dos n-alcanos em subestimar os valores de digestibilidade pode ser explicada pela menor taxa de recuperação fecal do indicador interno (n-alcano de cadeia ímpar natural da forragem) em relação ao externo (n-alcano sintético de cadeia par, fornecido). Segundo Dove \& Mayes (1991), aproximadamente 95\% dos alcanos de cadeia ímpar encontram-se na fase sólida da dieta, enquanto $40 \%$ dos $n$-alcanos fornecidos (indicador externo) estão associados à fase líquida, o que pode explicar, em parte, a diferença nas taxas de recuperação fecal entre os n-alcanos natural (forragem) e administrado (sintético).

Com base nestes fatos, pode-se especular que a acurácia na estimativa do consumo por meio da técnica dos n-alcanos depende também da forragem avaliada, pois a qualidade da fibra interfere na taxa de passagem. Alimentos com fibra de baixa qualidade (pouco degradável) tendem a maior tempo de retenção no rúmen. Maior tempo de retenção das partículas no trato digestório poderia ocasionar maior absorção do n-alcano natural (que estaria associado à fase sólida da dieta) em relação ao n-alcano fornecido (que, associado à fase líquida, tenderia a passar mais rapidamente pelo trato), promovendo menor recuperação fecal do n-alcano natural (forragem) em relação ao administrado.

Piasentier et al. (1995) avaliaram os dois métodos (n-alcanos versus $\mathrm{Cr}_{2} \mathrm{O}_{3}$ ) utilizando ovinos em pastejo e em gaiolas de estudos metabólicos. Nos animais em confinamento, comparando os resultados de consumo obtidos pelas técnicas aos valores medidos (reais), a metodologia do $\mathrm{Cr}_{2} \mathrm{O}_{3}$ /DIVMS superestimou o consumo em 5,5\%, enquanto o par $\mathrm{C}_{31}: \mathrm{C}_{32}$ subestimou estes valores em 3\%. No ensaio com animais em pastejo, os valores de consumo obtidos pela técnica do $\mathrm{Cr}_{2} \mathrm{O}_{3}$ /DIVMS foram $16,6 \%$ superiores aos estimados pelos n-alcanos. Neste ensaio, os autores não puderam determinar o indicador mais adequado, justamente pelo desconhecimento dos valores reais de consumo em condições de pastejo. 
Malossini et al. (1996) também estudaram as duas metodologias em vacas em lactação sob pastejo e encontraram, pela técnica do $\mathrm{Cr}_{2} \mathrm{O}_{3}$ /DIVMS, valores 5,1\% maiores que os obtidos com os n-alcanos. No entanto, ressaltaram que ambas as metodologias podem ser consideradas adequadas, ficando a critério do pesquisador a escolha daquela a ser utilizada.

Em vacas em lactação mantidas em pastagem de capimquicuio (Pennisetum clandestinum) e utilizando o par de n-alcanos $\mathrm{C}_{33}: \mathrm{C}_{32}$, Reeves et al. (1996) estimaram CMS de $12,6 \mathrm{~kg} /$ dia de MS, próximo ao predito nas tabelas de exigências (12,3 kg/dia de MS).

Neste trabalho, o par de n-alcanos $\mathrm{C}_{35}$ : $\mathrm{C}_{36}$ não foi utilizado na estimativa do CMS por dois motivos: 1) a concentração do $\mathrm{C}_{35}$ no capim-elefante estava muito próxima do mínimo de $50 \mathrm{mg} / \mathrm{kg}$ de MS recomendado por Laredo et al. (1991) para que um n-alcano possa ser utilizado como indicador interno de digestibilidade; 2) em vários cromatogramas das amostras de fezes, o n-alcano $\mathrm{C}_{36}$ foi encontrado em quantidades inferiores à esperada, ou não foi detectado (traço). No entanto, as concentrações dos n-alcanos $\mathrm{C}_{32} \mathrm{e}$ $\mathrm{C}_{36}$ analisadas nas $\mathrm{CRC}$ corresponderam às especificadas pelo fabricante, descartando a possibilidade de erro na extração e/ou leitura do $\mathrm{C}_{36}$ ou de sua eventual ausência na cápsula. Esta observação também foi relatada por Molina et al. (2004), que ressaltaram a importância do estudo do destino destes compostos no trato digestório dos animais, pois, segundo eles, algumas espécies de bactérias são capazes de utilizar n-alcanos em ambientes anaeróbicos. Além disso, de acordo com Ohajuruka \& Palmquist (1991), o rúmen seria o principal local de desaparecimento dos n-alcanos, justificando suas recuperações fecais incompletas.

Os valores de produção fecal estimados pelos diferentes indicadores foram avaliados pelo teste " $t$ " de Student para dados emparelhados e apresentaram diferença significativa ( $(P<0,05)$, com média de $0,27 \mathrm{~kg} /$ dia de MS de fezes. O óxido de cromo possibilitou estimar valores de produção fecal menores $(3,9 \mathrm{~kg} / \mathrm{dia}$ de MS) que os obtidos a partir do n-alcano $\mathrm{C}_{32}$ (4,2 kg/dia de MS). Soares et al. (2004) relataram valor médio de produção fecal, estimado com o óxido de cromo, $3,7 \mathrm{~kg} / \mathrm{animal} / \mathrm{dia}$ de MS para vacas Holandês x Zebu em lactação recebendo, no cocho, capim-elefante com 30 dias de idade de corte.

Os valores médios da DIVMS e os coeficientes de digestibilidade estimados pelos n-alcanos $\mathrm{C}_{31}$ e $\mathrm{C}_{33}$ foram, respectivamente, de 64,4 e 67,3; de 46,2 e 45,6; e de 51,3 e $51,3 \%$ para os meses de março e abril. Lopes et al. (2004), em estudo com capim-elefante manejado em sistema rotacionado, relataram valores de DIVMS de 61,3 e 60,8\% para os meses de março e abril, respectivamente.
Em vacas não-lactantes alimentadas com dieta à base de feno de alfafa e concentrado, Ohajuruka \& Palmquist (1991) observaram que o valor de $65,6 \%$ obtido para a digestibilidade in vivo da MS foi mais próximo ao estimado pelo indicador interno cinza insolúvel em ácido $(65,8 \%)$ em relação ao obtido pelo n-alcano $\mathrm{C}_{31}(55,6 \%)$. Os autores atribuíram essa diferença às recuperações fecais de ambos os indicadores, de 102 e 78,1\% para CIA e $\mathrm{C}_{31}$, respectivamente.

Oliveira et al. (2000), analisando o potencial do n-alcano $\mathrm{C}_{35}$ na estimativa da digestibilidade da MS e MO, compararam os valores estimados pelo n-alcano àqueles obtidos in vivo e relataram que o $\mathrm{C}_{35}$ subestimou a DMS e a DMO em 4,7 e $4,4 \%$, respectivamente.

A estimativa de maiores valores de produção fecal, associada à subestimativa do coeficiente de digestibilidade, é característica da metodologia dos n-alcanos (Astigarraga, 1997). Portanto, a aplicação da técnica objetivando estimar isoladamente valores de produção fecal ou de digestibilidade pode não ser recomendada, por estimar o CMS com base na premissa de que os erros associados às recuperações fecais incompletas dos n-alcanos de cadeia ímpar e de cadeia par são anulados na equação, sendo necessário que as taxas de recuperação sejam semelhantes.

A metodologia do $\mathrm{Cr}_{2} \mathrm{O}_{3}$ /DIVMS constitui técnica barata e de análise relativamente fácil. As cápsulas de liberação controlada (CRC) que contêm os n-alcanos possuem custo de, aproximadamente, US $\$ 30,00 /$ unidade. A metodologia dos n-alcanos apresentou custo, aproximadamente, 15 vezes maior que o da metodologia do $\mathrm{Cr}_{2} \mathrm{O}_{3}$ /DIVMS. Além disso, os n-alcanos são determinados por meio de cromatografia gasosa e os procedimentos para extração/purificação são complexos, envolvendo muitas etapas que aumentam as oportunidades para ocorrência de erros analíticos.

\section{Conclusões}

A metodologia do óxido de cromo/digestibilidade in vitro apresenta como vantagens a relativa simplicidade dos procedimentos analíticos e o baixo custo.

Em situações em que a disponibilidade de recursos financeiros não são restrição, a metodologia dos n-alcanos pode ser utilizada na estimativa do consumo de MS de pastagem, sendo recomendado, neste caso, o par $\mathrm{C}_{33}: \mathrm{C}_{32}$. Em condições em que a pastagem é formada por várias espécies forrageiras e em que é necessária a estimação do consumo diferenciado destas espécies ou em que o manejo intenso dos animais é fator de interferência importante, a metodologia dos n-alcanos pode ser a mais indicada. 


\section{Agradecimento}

Ao Dr. Dimas Estrásulas de Oliveira, pelas sugestões e pela colaboração.

\section{Literatura Citada}

AROEIRA, L.J.M. Estimativas de consumo de gramíneas tropicais. In: SIMPÓSIO INTERNACIONAL DE DIGESTIBILIDADE EM RUMINANTES, 1997, Lavras. Anais... Lavras: Universidade Federal de Lavras, 1997. p.127-163.

ASSOCIATION OF OFFICIAL ANALYTICAL CHEMISTS - AOAC. Official methods of analysis. 15.ed. Arlington: 1990. v.1, $1117 \mathrm{p}$.

ASTIGARRAGA, L. Técnicas para la medición del consumo de rumiantes em pastoreo. In: SIMPÓSIO SOBRE AVALIAÇÃO DE PASTAGENS COM ANIMAIS, 1997, Maringá. Anais... Maringá: Universidade Estadual de Maringá, 1997. p.1-23.

BERRY, N.R.; SCHEEDER, M.R.L.; SUTTER, F. et al. The accuracy of intake estimation based on the use of alkane controlledrelease capsules and faeces grab sampling in cows. Annales Zootechnie, v.49, p.3-13, 2000.

CÓSER, A.C.; MARTINS, C.E.; DERESZ, F. et al. Métodos para estimar a forragem consumível em pastagem de capimelefante. Pesquisa Agropecuária Brasileira, v. 38 , n.7, p.875-879, 2003.

DETMANN, E.; PAULINO, M.F.; ZERVOUDAKIS, J.T. et al. Cromo e indicadores internos na determinação do consumo de novilhos mestiços, suplementados, a pasto. Revista Brasileira de Zootecnia, v.30, n.5, p.1600-1609, 2001.

DOVE, H.; MAYES, R.W. Plant wax components: a new approach to estmating intake and diet composition in herbivores. Journal of Nutrition, v.126, n.1, p.13-26, 1996.

DOVE, H.; MAYES, R.W. The use of plant wax alkanes as marker substances in studies of the nutritional herbivores - A review. Australian Journal of Agricultural Research, v.42, n.6, p.913-952, 1991.

EUClIDES, V.P.; CARDoso, E.G.; MACEDO, M.C.C. et al. Consumo voluntário de Brachiaria decumbens cv. Basilisk e Brachiaria brizantha cv. Marandu sob pastejo. Revista Brasileira de Zootecnia, v.29, n.6, p.2200-2208, 2000 (supl.).

FONTES, C.A.A.; OLIVEIRA, M.A.T.; LANA, R.P. et al. Avaliação de indicadores na determinação da digestibilidade em novilhos. Revista da Sociedade Brasileira de Zootecnia, v.25, n.2, p.529-539, 1996.

GEDIR, J.V.; HUDSON, R.J. Estimating dry matter digestibility and intake in wapiti (Cervus elaphus canadensis), using the double n-alkane ratio technique. Small Ruminant Research, v.36, p.57-62, 2000 .

LAREDO, M.A.; SIMPSON, G.D.; MINSON, D.J. et al. The pontential for using $\mathrm{n}$-alkanes in tropical forages as marker for the determination of dry matter by grazing ruminants. Journal of Agricultural Science, v.117, n.3, p.355-361, 1991.

LOPES, F.C.F.; RODRIGUEZ, N.M.; AROEIRA, L.J.M. Uso dos nalcanos em estimativas de consumo de ruminantes sob pastejo: uma revisão. Veterinária Notícias, v.7, n.2, 2001.

LOPES, F.C.F.; AROEIRA, L.J.M.; RODRIGUEZ, N.M. et al. Efeito da suplementação e do intervalo de pastejo sobre a qualidade da forragem e consumo voluntário de vacas Holandês x Zebu em lactação em pastagem de capim-elefante. Arquivo Brasileiro de Medicina Veterinária e Zootecnia, v.56, n.3, p.355-362, 2004.

LOPES, F.C.F.; DERESZ, F.; RODRIGUEZ, N.M. et al. Disponibilidade e perdas de matéria seca em pastagem de capim-elefante (Pennisetum purpureum, Schumack) submetida a diferentes períodos de descanso. Arquivo Brasileiro de Medicina Veterinária e Zootecnia, v.55, n.4, p.454-460, 2003.
MALOSSINI, F.; BOVOLENTA, S.; PIASENTIER, C. et al. Comparison of n-alkanes and chromium oxide methods for estimating herbage intake by grazing dairy cows. Animal Feed Science and Technology, v.61, p.155-165, 1996.

MAYES, R.W.; LAMB, C.S.; COLGROVE, P.M. The use of dosed and herbage n-alkanes as markers for determination of herbage intake. Journal of Agriculture Science, v.107, n.1, p. $161-170,1986$.

MOLINA, D.O.; MATAMOROS, I.; PELL, A.N. Accuracy of estimates of herbage intake of lactating cows using alkanes: comparison of two types of capsules. Animal Feed Science and Technology, v.114, p.241-260, 2004.

NATIONAL RESEARCH COUNCIL - NRC. Nutrient requirements of dairy cattle. 7.ed.rev. Washington, D.C.: National Academy Press, 2001. 408p.

OHAJURUKA, O.A.; PALMQUIST, D.L. Evaluation of n-alkanes as digesta markers in dairy cows. Journal of Animal Science, v.69, n.4, p.1726-1732, 1991.

OLIVÁN, M.; OSORO, K. The effect of drying treatment on the nalkane analysis. Annales Zootechnie, v.44, p.238, 1995 (suppl.).

OLIVEIRA, D.E. Determinação de alcanos: manual de extração e análise cromatográfica em forragens, concentrados e fezes. Piracicaba: Fundação de Estudos Agrários "Luiz de Queiroz", 2004. 30p.

OLIVEIRA, D.E. Uso da técnica dos n-alcanos para medir o aporte de nutrientes através de estimativas do consumo de forragem em bovinos. Piraciaba: Escola Superior de Agricultura Luiz de Queiroz, 2003. 129p. Tese (Doutorado em Agronomia) Escola Superior de Agricultura Luiz de Queiroz, 2003.

OLIVEIRA, D.E.; PRATES, E.R.; PERALBA, M.C.R. Identificação e quantificação de n-alcanos presentes nas ceras de plantas forrageiras. Revista Brasileira de Zootecnia, v.26, n.5, p.881-886, 1997.

OLIVEIRA, D.E.; PRATES, E.R.; PERALBA, M.C.R. Digestibilidade determinada pelo método indireto usando o n-alcano $\mathrm{C}_{35}$. Revista Brasileira de Zootecnia, v.29, n.3, p.848-852, 2000.

PACIULLO, D.S.C.; AROEIRA, L.J.M.; DERESZ, F. et al. Disponibilidade de matéria seca e consumo de forragem de vacas em lactação em pastagem de capim elefante. In: REUNIÃO ANUAL DA SOCIEDADE BRASILEIRA DE ZOOTECNIA, 38., 2001, Piracicaba. Anais... Piracicaba: Sociedade Brasileira de Zootecnia, 2001. (CD-ROM).

PAIM-COSTA, M.L.; DERESZ, F.; CÓSER, A.C. et al. Avaliação da disponibilidade e composição bromatológica do capim-elefante (Pennisetum purpureum Schum., cv. Napier), sob pastejo usando vacas Holandês x Zebu, na época chuvosa. In: REUNIÃO ANUAL DA SOCIEDADE BRASILEIRA DE ZOOTECNIA, 41. 2004, Campo Grande. Anais... Campo Grande: Sociedade Brasileira de Zootecnia, 2004. (CD-ROM).

PIASENTIER, E.; BOVOLENTA, S.; MALOSSINI, F. et al. Comparison of n-alkanes or chromium oxide methods for estimation of herbage intake by sheep. Small Ruminant Research, v.18, p.27-32, 1995.

POND, K.R.; ELLIS, W.C.; MATIS, J.H.; DESWYSEN, A.G. Passage of chromium-mordanted and rare-earth-labeled fiber: time dosing kinetics. Journal of Animal Science, v.67, p.10201028,1989 .

REEVES, M.; FULKERSON, W.J.; KELLAWAY, R.C. A comparison of three techniques to determine the hebage intake of dairy cows grazing kikuyu (Pennisetum clandestinum) pasture. Australian Journal of Experimental Agriculture, v. 36, p.23-30, 1996.

UNIVERSIDADE FEDERAL DE VIÇOSA - UFV. SAEG - Sistemas para Análises Estatísticas e Genéticas. versão 8.0 - manual de instruções. Viçosa, MG: CPD/UFV, Divisão de Pesquisa e Desenvolvimento, 2000. 142p.

SOARES, J.P.G.; AROEIRA, L.J.M.; DERESZ, F. et al. Avaliação do consumo de vacas em lactação, medido em sistema "calangates" e estimado pelo óxido de cromo. In: REUNIÃO ANUAL 
DA SOCIEDADE BRASILEIRA DE ZOOTECNIA, 36., 1999, Porto Alegre. Anais... Porto Alegre: Sociedade Brasileira de Zootecnia, 1999. (CD-ROM).

SOARES, J.P.G.; BERCHIELLI, T.T.; AROEIRA, L.J.M. et al. Estimativa de consumo do capim-elefante (Pennisetum purpureum Schum.), fornecido picado para vacas lactantes utilizando a técnica do óxido crômico. Revista Brasileira de Zootecnia, v.33, n.3, p.811-820, 2004.

SOARES, J.P.G., BERCHIELli, T.T., AROEIRA, L.J.M. et al. Evacuação de vacas lactantes para determinação do rúmen fill induzido pelo capim-elefante (Pennisetum purpureum, Schum.) em 3 idades de corte. In: REUNIÃO ANUAL DA SOCIEDADE BRASILEIRA DE ZOOTECNIA, 37., 2000, Viçosa, MG. Anais... Viçosa, MG: Sociedade Brasileira de Zootecnia, 2000. (CD-ROM).

TILLEY, J.M.A.; TERRY, R.A. A two stages technique for the "in vitro" digestion of forage crops. Journal British of Grassland Society, v.18, n.1, p.104-111, 1963.
UNAL, Y.; GARNSWORTHY, P.C.; GORTON, P. The use of nalkanes for prediction of intake in dairy cows. Proceedings of the British Society of Animal Science, 1997. 137p.

Van DYNE, G.M.; TORELL, D.T. Development and use of esophageal fistula: a review. Journal of Range Management, v. 17, p.7-19, 1964.

VULICH, S.A.; HANRAHAN, J.P.; O'ORIORDAN, E.G. Pasture sampling for estimation of herbage intake using n-alkanes: evaluation of alternative sampling procedures. Irish Journal of Agriculture Food Research, v.32, p.1-11, 1993.

WILLIAMS, C.H.; DAVID, D.J.; IISMAA, O. The determination of chromic oxide in faeces samples by atomic absorption spectrophotometry. Journal of Agriculture Science, v. 59, n.3, p.381-385, 1962.

Recebido: 10/08/05 Aprovado: 23/01/06 